\title{
Einfluss einer Hormonersatz-Therapie auf die Hautalterung
}

H. Hönigsmann

Influence of Hormone Substitution on Intrinsic Skin Aging

\section{Zusammenfassung}

Der postmenopausale Östrogenabfall trägt maßgeblich zum intrinsischen Alterungsprozess der Haut bei. Wie mittlerweile mehrere Studien deutlich machen, wirkt ein Ausgleich dieses hormonellen Defizits durch eine systemische oder topische Substitution von Östrogen der Hautalterung entgegen. Um eine Aussage darüber zu treffen, welches Östrogen in welcher Konzentration am besten geeignet sein könnte, sind - vor allem unter Berücksichtigung potenzieller systemischer Nebenwirkungen weitere kontrollierte Studien erforderlich.

\section{Abstract}

Postmenopausal decrease of estrogen is an important process for intrinsic skin aging. Several studies show that a systemic or topical estrogen substitution is able to compensate these hormonal deficits and counteract skin aging. Further studies are necessary to evaluate which estrogen in which concentration is most suitable - mainly regarding potential systemic side effects.

\section{Einleitung}

Insbesondere in Anbetracht der wachsenden Zahl älterer Menschen ist es Aufgabe der kosmetischen Dermatologie, die physiologische Funktion und auch das äußere Erscheinungsbild der Haut im fortgeschrittenen Lebensalter weitest möglich zu erhalten. Denn mit zunehmendem Alter verliert die Haut an Festigkeit, sie wird trockener, ungleichmäßiger pigmentiert und es bilden sich Falten. Im Rahmen dieser alterstypischen Prozesse vermindert sich die Dicke der Haut und es kommt zur Reduktion und Dysfunktion verschiedener zellulärer Komponenten (Tab.1, Abb.1). Parallel dazu werden verschiedene Funktionen der Haut beeinträchtigt:

- Hauterneuerung,

- Barrierefunktion,

- Schweißproduktion,

- Sebumproduktion,

- Thermoregulation sowie

- vaskuläre und immunologische Reaktionen.
Während die extrinsische Hautalterung (Photoaging) primär durch die kumulative UV-Dosis determiniert wird, hängt die intrinsische Hautalterung neben der genetischen Disposition vor allem von metabolischen und hormonellen Störungen ab. Zentrale Rolle bei der hormonellen Regulation der Alterungsprozesse der Haut bzw. der Hautanhangsorgane spielen Östrogene und Androgene (Tab. 2).

Androgene stimulieren die Talgdrüsen und sind maßgeblich an der Ausbildung von Seborrhö und Akne beteiligt. Darüber hinaus ist auch der Haarzyklus hormongesteuert: Androgene bewirken eine Reduktion der Anagenphase und spielen somit eine relevante Rolle bei der androgenetischen Alopezie. Im Unterschied dazu inhibieren Östrogene die Funktion der Talgdrüsen, sie verlängern die Wachstumsphase der Haare und besitzen einen anti-atrophischen Effekt auf epitheliales, kollagenes und elastisches Gewebe. Der Östrogen-Rezeptor, der ein Indikator dafür ist, dass die Zel-

Institutsangaben

Abteilung für Spezielle Dermatologie und Umweltdermatosen, Allgemeines Krankenhaus der Stadt Wien, Universität Wien, Wien/Österreich

Prof. Dr. Herbert Hönigsmann · Universität Wien · Abteilung für Spezielle Dermatologie und Umweltdermatosen · Allgemeines Krankenhaus der Stadt Wien · Währinger Gürtel 18 - 20 · 1090 Wien . Österreich·E-mail: herbert.hoenigsmann@akh-wien.ac.at 
Tab. 1 Reduktion und Dysfunktion der zellulären Komponenten im Rahmen der Hautalterung

\begin{tabular}{lll}
\hline Epidermis & Dermis & Hautanhangsgebilde \\
\hline verminderte Dicke & Atrophie & $\begin{array}{l}\text { Depigmentierung } \\
\text { der Haare }\end{array}$ \\
\hline $\begin{array}{l}\text { veränderte Größe und } \\
\text { Form der Zellen }\end{array}$ & $\downarrow$ Fibroblasten & Haarverlust \\
\hline Zellkernveränderungen & $\downarrow$ Blutgefäße & $\begin{array}{l}\text { Konversion vom } \\
\text { terminalen zum } \\
\text { Vellushaar }\end{array}$ \\
$\begin{array}{l}\downarrow \text { Melanozyten } \\
\downarrow \text { Langerhans Zellen }\end{array}$ & $\begin{array}{l}\text { abnormale Nerven- } \\
\text { zellendigungen }\end{array}$ & \\
\hline
\end{tabular}

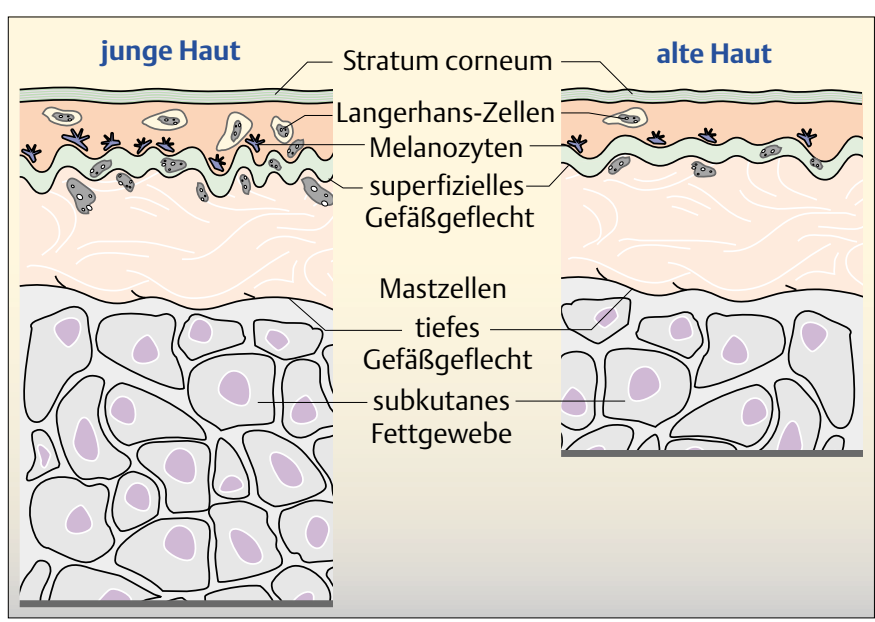

Abb. 1 Im Rahmen der Hautalterung vermindert sich die Dicke der Haut und es kommt zur Reduktion und Dysfunktion verschiedener zellulärer Komponenten.

Tab. 2 Hormone und ihre Funktionen innerhalb der Haut und den Haaren

\begin{tabular}{ll}
\hline Androgene & Östrogene \\
\hline $\begin{array}{l}\text { Stimulation der Talgdrüsen } \\
\text { (Seborrhö, Akne) }\end{array}$ & Inhibition der Talgdrüsen \\
\hline $\begin{array}{l}\text { Haarzyklus: Reduktion der Anagen- } \\
\text { phase (androgenetische Alopezie) }\end{array}$ & $\begin{array}{l}\text { Haarzyklus: Verlängerung der } \\
\text { Wachstumsphase }\end{array}$ \\
& $\begin{array}{l}\text { Anti-atrophischer Effekt auf epi- } \\
\text { theliale, kollagene und elastische } \\
\text { Fasern } \\
\text { Effekt auf die Hautalterung? }\end{array}$ \\
\hline
\end{tabular}

len auf Östrogene ansprechen, befindet sich auf Keratinozyten, Fibroblasten, Sebozyten und Gefäßendothelien.

Der postmenopausale Östrogenmangel wirkt sich auf das hauteigene Kollagen sowie die elastischen Fasern aus und trägt auf diesem Weg maßgeblich zur endogenen Hautalterung bei (Abb. 2). Aus Tierversuchen weiß man, dass Östrogene die Polymerisie-

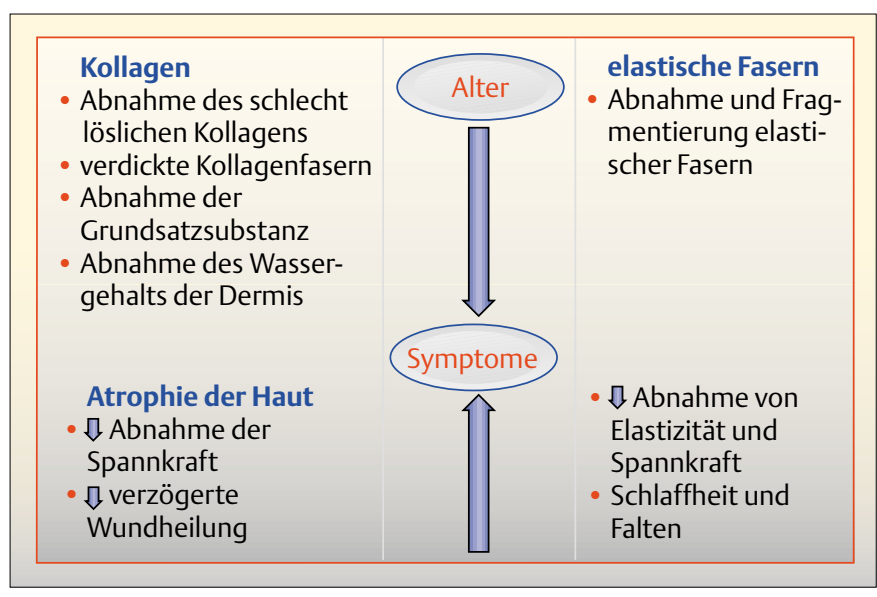

Abb. 2 Der postmenopausale Östrogenmangel verursacht Veränderungen am Kollagen und den elastischen Fasern und löst so die Symptome der Hautalterung aus.

rung der löslichen niedermolekularen Form zur unlöslichen höhermolekularen Form stimulieren. Zugleich hemmen Östrogene den Abbau von Kollagen, so dass die Halbwertszeit um mehr als $100 \%$ verlängert wird. Nach der Menopause nimmt der Kollagengehalt signifikant um bis zu 30\% ab und auch die Struktur der Kollagenfasern verändert sich: Sowohl der Umsatz, die Kreuzvernetzung, der Glykosamingehalt als auch der dermale Wassergehalt sind reduziert. Viele Symptome der Hautalterung sind mit der Postmenopause assoziiert. So wird die Haut parallel zu dem Absinken der endogenen Hormonspiegel trockener, die Elastizität und Festigkeit nimmt ab und es kommt zu einem charakteristischen „Absacken“ der Haut im Gesicht und am Körper. Eine Östrogensubstitution könnte diesem hautspezifischen Alterungsprozess demnach möglicherweise entgegenwirken.

Bei der für postmenopausale Frauen charakteristischen urogenitalen Atrophie wird eine topische Östrogensubstitution bereits seit vielen Jahrzehnten erfolgreich eingesetzt. Da Östrogene eine anti-atrophische und kollagenstimulierende Wirkung sowie einen Effekt auf die elastischen Fasern der Haut besitzen, wurde darüber hinaus in mehreren Studien der Frage nachgegangen, ob eine systemische oder eine topische Hormonsupplementation einen Anti-Aging-Effekt besitzt.

\section{Epidermale und dermale Dicke unter HRT signifikant erhöht} Bereits Anfang der 70er-Jahre untersuchte man im Rahmen einer kontrollierten Studie die Auswirkung einer Hormonersatz-Therapie (HRT) auf die Haut [1,2]. An der Studie nahmen Frauen drei Monate nach der Menopause teil, bei denen die epidermale und dermale Dicke der Haut bereits signifikant reduziert war. Bereits drei bis sechs Monate nach Beginn der hormonellen Substitution erhöhte sich sowohl die epidermale als auch die dermale Dicke der Haut in signifikantem Ausmaß.

Weitere Bestätigung der positiven Wirkung einer HormonersatzTherapie auf die Hautalterung brachte einige Jahre später die National Health and Nutrition Examination Survey (NHANES I) [3]. In dieser epidemiologischen Erhebung an 3875 postmenopausalen Frauen im durchschnittlichen Alter von 61,6 Jahren wurde unter anderem der Effekt einer systemischen Hormonersatz- 
Therapie auf die Haut untersucht. Die Ergebnisse belegen, dass eine systemische Gabe von Östrogenen die Haut sowohl vor Falten als auch vor der charakteristischen postmenopausalen Trockenheit schützt. So nahmen bei den Frauen unter der HRT:

- die Hauttrockenheit um 25\% und

- die Falten um 30\% ab.

Die Autoren folgern, dass eine postmenopausale Östrogensubstitution einen potenziellen Benefit bei der menopauseassoziierten Hautalterung bietet.

Hautalterung unter systemischer Östrogentherapie verzögert Genaueren Aufschluss brachte eine eigene Studie, an der 24 postmenopausale Frauen im durchschnittlichen Alter von 55 Jahren teilnahmen [4]. Die Patientinnen wurden auf drei Studienarme verteilt und über sechs Monate nach folgendem Therapieschema behandelt:

- Estradiol ${ }^{\circledR}$ (transdermales System, TSS 50, $50 \mu \mathrm{g} 17 \beta \mathrm{E} 2$ )

- Estraderm ${ }^{\circledR}$ (TTS 50+ Progesteron 0,4 mg vaginales Suppositorium)

- Progynova ${ }^{\circledR}$ (Östrogen 2 mg p.o. + Progesteron 0,4 mg vaginales Suppositorium).

Als interne Kontrolle diente eine vierte Gruppe von drei Patientinnen, die nicht behandelt wurden.

Um den Einfluss der HRT auf die Hautalterung möglichst genau zu untersuchen, wurde sowohl an sonnenexponierten Hautarealen wie Gesicht und Dekolletee als auch an sonnengeschützten Bereichen wie der inneren Oberarmseite folgende Parameter untersucht:

- Hautoberflächenlipide (Sebumeter ${ }^{\circledR}$ SM 810)

- epidermale Hydratation (Corneometer CM 820)

- Hautelastizität (Dermaflex A ${ }^{\circledR}$ )

- Hautdicke (Osteoson ${ }^{\circledR}$ DIII)

- zusätzlich wurde alle drei Monate der Hormonspiegel (E2, FSH, Progesteron) bestimmt.

Die subjektiven Angaben der Patientinnen bestätigen, dass sich unter der HRT sowohl die Trockenheit der Haut vermindert als auch die Festigkeit der Haut steigert. Die in Abb. 3 zusammengefassten Ergebnisse der instrumentellen Messungen belegen darüber hinaus in allen drei Behandlungsgruppen nahezu durchgängig einen positiven Effekt auf den Feuchtigkeits- und Lipidgehalt sowie die hauteigene Elastizität und die Hautdicke. Während der Lipidgehalt unter einer Östrogen-Monotherapie aufgrund des sebumsuppressiven Effekts der Östrogene absank, stieg der Lipidgehalt unter einer kombinierten HRT (Östrogen + Progesteron) an. Maßgeblich ist hierbei der stimulierende Effekt von Progesteron auf die hauteigenen Talgdrüsen. Parallel dazu verbesserte sich die Hautelastizität, die epidermale Hydratation und die Dicke der Haut an sämtlichen Stellen inklusive der UVexponierten Hautareale.

Obwohl eine verzögerte Hautalterung nicht das primäre Ziel einer HRT ist, scheint sich die Hormonbehandlung auch hinsichtlich des Hautbildes auszuzahlen. Durch die gestiegene Elastizität und den erhöhten Feuchtigkeitsgehalt der Haut verbesserte die Hormonbehandlung das jugendliche Aussehen der Frauen; aller-

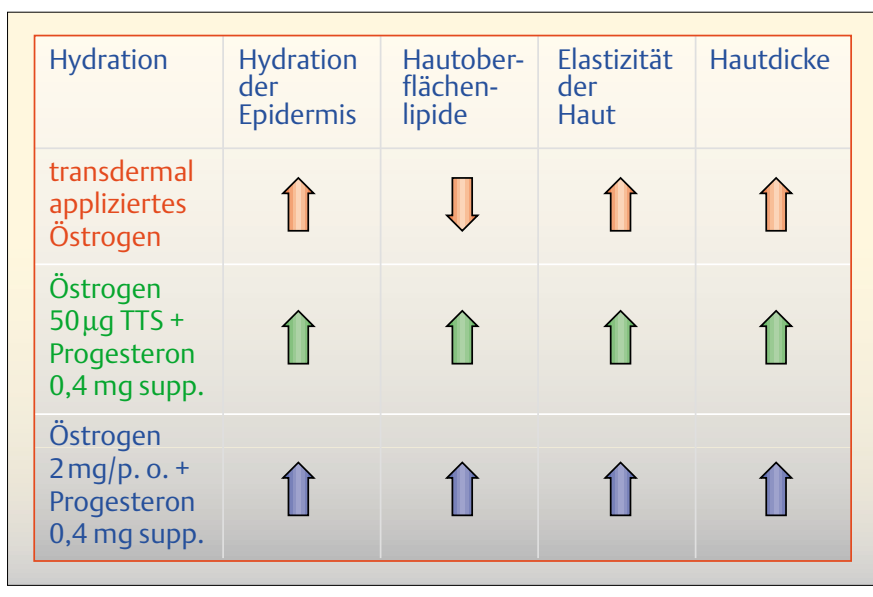

Abb. 3 Einfluss der HRT auf die Hautalterung.

dings führte die kurzfristige Behandlung nicht zu einem Effekt hinsichtlich der Faltenbildung.

Die Ergebnisse sprechen dafür, dass ein hormonelles Defizit bei der körpereigenen Hautalterung eine größere Rolle spielt als bislang angenommen und dass auch Frauen mit einer UV-induzierten Hautalterung von einer HRT profitieren. Möglicherweise besitzt eine Hormontherapie einen positiven Langzeiteffekt und ist in der Lage, den Alterungsprozess zu verzögern.

\section{Vergleichbare Ergebnisse unter topischer Östrogenapplikation}

Um systemische Nebenwirkungen zu vermeiden, ging eine weitere Studie in doppelblindem und randomisiertem Design der Frage nach, ob eine topische Östrogenapplikation vergleichbare Effekte nach sich zieht [5]. Eingeschlossen waren 58 postmenopausale Frauen zwischen 52 und 70 Jahren, die über 24 Wochen entweder mit konjugierten Östrogenen (Premarin ${ }^{\circledR}$ Creme, $1 \mathrm{~g} / \mathrm{Tag}$ ) oder mit Plazebo behandelt wurden. Den Ergebnissen zufolge bewirkt eine topische Östrogenapplikation:

- einen signifikanten Anstieg der Hautdicke

- eine signifikante Reduktion der Falten

- keine hormonellen Nebenwirkungen.

Auch eine Studie, in der peri- oder postmenopausale Frauen mit Östradiol (0,01\%; $1 \mathrm{~g} / \mathrm{Tag})$ bzw. Östriol (0,3\%; $1 \mathrm{~g} / \mathrm{Tag})$ erhielten, spricht für eine positive Wirkung topisch applizierter Hormone auf die Hautalterung [6]. Die Frauen in den beiden Behandlungsgruppen ( $n=28$ bzw. $n=30$ ) wurden über sechs Monate behandelt und neben einer gynäkologischen Untersuchung nach drei bzw. sechs Monaten einmal monatlich Prolaktin, FSH (Follikel stimulierendes Hormon) und Östradiol bestimmt. Am Ende der Studie hatten sich in beiden Gruppen verschiedene, für die Hautalterung relevante Parameter verbessert, wobei die Effekte des topisch applizierten Östriols sowohl hinsichtlich des Wirkungseintritts als auch der Wirkstärke etwas ausgeprägter waren. In keiner der beiden Behandlungsgruppen wurden hormonelle Nebenwirkungen festgestellt. 
Tab. 3 Topisches Östradiol versus Östriol bei der Hautalterung - Ergebnisse der Corneometrie und Profilometrie

\begin{tabular}{lllll}
\hline Corneometrie (Einheiten*) & $\mathbf{n}$ & Ausgangswert & Endwert & P \\
Östradiol & 20 & $66 \pm 27$ & $79 \pm 14$ & $\mathrm{p}>0,05$ (ns) \\
Östriol & 20 & $66 \pm 26$ & $78 \pm 16$ & $\mathrm{p}>0,5$ (ns) \\
Profilometrie (RZ-D in $\mu \mathbf{m}^{*}$ ) & $\mathbf{n}$ & Ausgangswert & Endwert & $\mathbf{P}$ \\
Östradiol & 21 & $50 \pm 18$ & $41 \pm 16$ & $\mathrm{p}=0,002(\mathrm{~s})$ \\
Östriol & 21 & $48 \pm 20$ & $38 \pm 22$ & $\mathrm{p}=0,0005$ (hs) \\
\hline
\end{tabular}

* Einheit $=0,02 \mathrm{ng} / \mathrm{cm}^{2}$ Wassergehalt des Stratum corneums; RZ-D = Arithmetisches Mittel für die maximale Rauheit.

Tab. 4 Topisches Östradiol versus Östriol bei der Hautalterung Behandlungsergebnisse

\begin{tabular}{|c|c|c|c|c|}
\hline & $\begin{array}{l}\quad \text { Östradiol ( } \\
\quad n=30 \\
\text { Verbesserung } \\
\text { (\%) }\end{array}$ & $\begin{array}{l}0,01 \%) \\
\text { Wirkungs- } \\
\text { eintritt } \\
\text { nach Wochen }\end{array}$ & $\begin{array}{l}\quad \begin{array}{l}\text { Östriol (0, } \\
n=28 \\
\text { Verbesserung } \\
\text { (\%) }\end{array}\end{array}$ & $\begin{array}{l}\text {,3\%) } \\
\text { Wirkungs- } \\
\text { eintritt } \\
\text { nach Wochen }\end{array}$ \\
\hline $\begin{array}{l}\text { Hautelastizität/ } \\
\text { Festigkeit }\end{array}$ & 100 & 13 & 96 & 11 \\
\hline Hydratation & 100 & 9 & 96 & 8 \\
\hline Faltentiefe & 87 & 16 & 89 & 17 \\
\hline
\end{tabular}

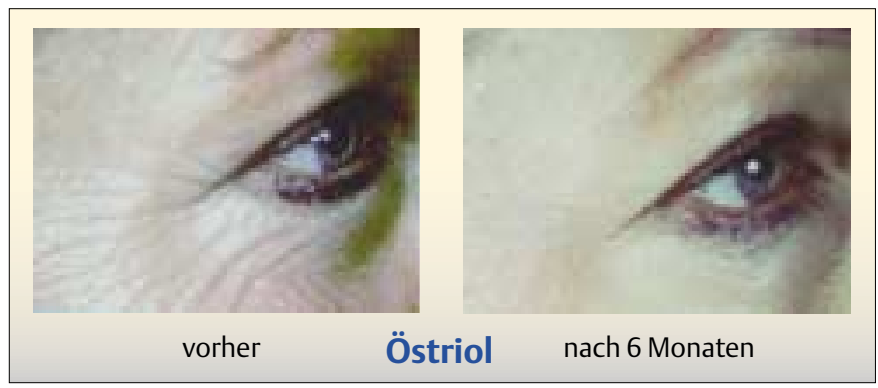

Abb. 4 Unter der topischen Östriolapplikation vermindert sich die Faltentiefe.

\section{Welches Östrogen eignet sich zur topischen Applikation?}

$\mathrm{Zu}$ einem vergleichbaren Ergebnis kam eine kontrollierte Studie unter Einschluss von 59 postmenopausalen Frauen, deren Haut bereits Zeichen einer Hautalterung zeigte [7]. Die Frauen wurden mit Östradiol ( $0,001 \%)$ bzw. mit Östriol $(0,3 \%)$ behandelt und mit Hilfe der Corneometrie und der Profilometrie eine Reihe dermatologischer Parameter erfasst (Tab. 3). Zusätzlich wurde anhand von Hautbiopsien immunohistochemisch der Gehalt an Kollagen I und III ermittelt. Den Ergebnissen zufolge waren nach sechs Monaten in beiden Behandlungsgruppen sowohl die Elastizität, die Festigkeit als auch die Hydratation der Haut signifikant verbessert (Tab. 4). Sämtliche der untersuchten Parameter verbesserten sich um etwa $100 \%$. Darüber hinaus nahmen in beiden Gruppen die Faltentiefe und auch die Porengröße signifikant zwischen 61 und $100 \%$ ab.

Den immunhistochemischen Untersuchungen zufolge waren in beiden Behandlungsgruppen am Ende der Studie signifikant mehr Kollagenfasern und Kollagen Typ III vorhanden. Dies erklärt die erhöhte Festigkeit und die geringere Faltentiefe der Haut von den mit Östrogen behandelten Frauen (Abb. 4).

Der Effekt von topisch appliziertem Östriol war sowohl hinsichtlich des Ausmaßes als auch des Wirkungseintritts leicht überlegen. Aufgrund der stärkeren Absorption erscheinen systemische Nebenwirkungen unter Östradiol zumindest theoretisch möglich. Östriol wird zwar weniger stark absorbiert, besitzt jedoch eine höhere Affinität zur Epidermis. Weder aufgrund des klinischen Bildes noch aufgrund des hormonellen Monitorings ergaben sich Hinweise auf Nebenwirkungen.

Fazit

Topisch applizierte Östrogene vermindern die Faltentiefe, bewirken einen Anstieg von Kollagen Typ III und erhöhen sowohl die Hydratation, die Festigkeit als auch die Dicke der Haut. Damit eröffnen topisch applizierte Östrogene einen viel versprechenden präventiven bzw. kurativen Ansatz zur Behandlung der Hautalterung. Um das Risiko systemischer hormoneller Effekte zu minimieren, sollten allerdings sowohl die Konzentration als auch das Ausmaß der Applikation klar definiert werden. Erst weitere doppelblinde Studien erlauben eine Aussage darüber, welches Östrogen in welcher Konzentration am besten geeignet sein könnte.

\section{Literatur}

${ }^{1}$ Punnonen R. Effect of castration and peroral estrogen therapy on the skin. Acta Obstet Gynecol Scand Suppl 1972; 21: 3-44

2 Brincat M, Moniz CJ, Studd JW, Darby A, Magos A, Emburey G, Versi E. Long-term effects of the menopause and sex hormones on skin thickness. Br J Obstet Gynaecol 1985; 92: 256

${ }^{3}$ Dunn LB, Damesyn M, Moore AA, Reuben DB, Greendale GA. Does estrogen prevent skin aging? Results from the First National Health and Nutrition Examination Survey (NHANES I). Arch Dermatol 1997; 133: $339-342$

${ }^{4}$ Sator PG, Schmidt JB, Sator MO, Huber JC, Hönigsmann H. The influence of hormone replacement therapy on skin ageing: a pilot study. Maturitas 2001; 39: $43-55$

${ }^{5}$ Creidi P, Faivre B, Agache P, Richard E, Haudiquet V, Auvanet JP. Effect of a conjugated oestrogen (Premarin) cream on ageing facial skin. A comparative study with a placebo cream. Maturitas 1994; 19: $211-223$

${ }^{6}$ Schmidt JB, Binder M, Macheiner W, Kainz C, Gitsch B, Biegelmayer C. Treatment of skin ageing symptoms in perimenopausal females with estrogen compounds. A pilot study. Maturitas 1994; 20: 25-30

${ }^{7}$ Schmidt JB, Binder M, Demschik G, Biegelmayer C, Reiner A. Treatment of skin aging with topical estrogens. Int J Dermatol 1996; 35: 669-674 